\title{
SOME RESULTS ON BLOW UP FOR SEMILINEAR PARABOLIC PROBLEMS
}

\section{M.A. HERRERO* AND J.J.L. VELÁZQUEZ*i}

Abstract. We discuss on recent results concerning the asymptotics near blow-up of nonnegative solutions of

$$
\begin{aligned}
& u_{t}=u_{x x}+f(u) \quad ; \quad-\infty<x<\infty, \quad t>0 \\
& u(x, 0)=u_{0}(x) \quad ; \quad-\infty<x<\infty,
\end{aligned}
$$

where $f(u)=u^{p}$ with $p>1$ or $f(u)=e^{u}$, and $u_{0}(x)$ is continuous, nonnegative and bounded.

AMS(MOS) subject classifications. 35B40, 35K55, 35K57

Key words and phrases: Semilinear parabolic problems, blow up, asymptotic behaviour of solutions.

1. Introduction. We shall consider one-dimensional equations of the type

$$
\begin{aligned}
& u_{t}=u_{x x}+f(u), \\
& \text { where } f(u)=u^{p} \quad(p>1) \quad \text { or } \quad f(u)=e^{u},
\end{aligned}
$$

which arise in many problems in continuum mechanics. For instance, combustion of a one-dimensional solid fuel is described by the set of equations ( $\mathrm{cf}[\mathrm{BE}]$ )

$$
\begin{aligned}
& T_{t}=T_{x x}+\delta \varepsilon c \exp \left(\frac{T-1}{\varepsilon T}\right) \\
& c_{t}=-\varepsilon \Gamma \delta c \exp \left(\frac{T-1}{\varepsilon T}\right),
\end{aligned}
$$

where $T$ and $c$ represent respectively the fuel temperature and concentration, and $\delta, \Gamma, \varepsilon$ are (positive) physical constants. Typically, $\varepsilon$ represents the inverse of the activation energy, and if we assume $0<\varepsilon \ll 1$, and look for solutions in the form

$$
T=1+\varepsilon u+\cdots \quad, \quad c=1-\varepsilon C_{1}+\cdots
$$

one is led to

$$
u_{t}=u_{x x}+\delta e^{u} \quad, \quad\left(C_{i}\right)_{t}=\Gamma \delta e^{u},
$$

and the first equation reduces to (1.1) with $f(u)=\delta e^{u}$. 
Solutions of (1.1) are known to develop singularities in finite time, regardless of the smoothness of their initial and boundary conditions. For spatially homogeneous (i.e., space independent) solutions, this is readily seen by integrating the first order PDE obtained by dropping the diffusion term $u_{x x}$ in (1.1). In the case of general solutions, it is interesting to understand the role of diffusion in the onset and character of singularities. We will say that a nonnegative solution $u(x, t)$ of (1.1) blows up at a time $T<+\infty$ if

$$
\lim _{t \uparrow T} \sup (\sup u(x, t))=+\infty
$$

Assume that $u(x, t)$ blows up at $t=T$. We then say that $x_{0}$ is a blow-up point of $u$ if there exist sequences $\left\{x_{n}\right\},\left\{t_{n}\right\}$, such that $\lim _{n \rightarrow \infty} x_{n}=x_{0}, \lim _{n \rightarrow \infty} t_{n}=T$, and

$$
\lim _{n \rightarrow \infty} u\left(x_{n}, t_{n}\right)=+\infty
$$

The set of blow-up points of $u$ is usually referred to as the blow-up set. sufficient conditions for blow up have been extensively discussed in the literature (cf. $[\mathrm{Fu}],[\mathrm{Le} 1],[\mathrm{AW}],[\mathrm{W}],[\mathrm{L}],[\mathrm{Be}], \ldots)$. Furthermore, the structure of the blow-up set, and the asymptotic behaviour of solutions near blow-up points have received considerable interest (cf $[\mathrm{GP} 2],[\mathrm{GK} 1],[\mathrm{GK} 2],[\mathrm{FM}],[\mathrm{CF}],[\mathrm{BBE}],[\mathrm{CM}],[\mathrm{FK}], \ldots$ ). These are precisely the questions we want to discuss upon herein. In doing so, we shall follow the approach adopted in $[\mathrm{HV} 1],[\mathrm{HV} 2],[\mathrm{HV} 3]$. The reader is referred to these papers for additional details.

Consider first the question of the asymptotics near blow-up, and for definiteness, let us consider solutions of the Cauchy problem

$$
\begin{aligned}
& u_{t}=u_{x x}+f(u) \quad ; \quad x \in \mathbb{R}, \quad t>0, f \text { as in (1.1), } \\
& u(x, 0)=u_{0}(x) \quad ; \quad u_{0} \quad \text { continuous, nonnegative and bounded. }
\end{aligned}
$$

Assume that $u(x, t)$ blows up in a finite time (in view of the results in $[\mathrm{Fu}],[\mathrm{AW}], \ldots$ this is certainly the case if $u_{0}(x) \not \equiv 0$ and $f(u)=e^{u}$ or $f\left(u=u^{p}\right.$ with $1<p \leq 3$, or if $u_{0}(x)$ is large enough when $p>3$ in this last case). Suppose also that $x=0$ is a blow up point for $u$. Then a basic asymptotic result states that

$$
\text { if } f(u)=u^{p} \quad(p>1), \lim _{t \uparrow T}(T-t)^{\frac{1}{p-1}} u\left(y(T-t)^{1 / 2}, t\right)=(p-1)^{-\frac{1}{p-1}}
$$

uniformly on sets $|y| \leq C$ with $C>0$,

$$
\text { if } f(u)=e^{u}, \lim _{t \uparrow T}\left(u\left(y(T-t)^{1 / 2}, t\right)+\log (T-t)\right)=0
$$


$u(x, t)$ behaves near blow up as the explicit self-similar solutions

$$
\begin{aligned}
& \bar{u}(x, t)=(p-1)(T-t))^{-\frac{1}{p-1}} \\
& \widetilde{u}(x, t)=-\log (T-t)
\end{aligned}
$$

along suitable backward parabolae centered at blow-up points.

A question which naturally arises is how to obtain more detailed expansions in larger regions, and in particular, what is the space profile of the singularity at $t=T$ (the so-called final-time analysis). It will turn out that, while first order asymptotics as that in (1.3) is remarkably uniform, higher-order expansions will depend on the concrete shape of the initial value -or rather, on its nodal properties (cf. Theorem 1, 2 and 3 below). To describe precisely the relevant results, we shall specialize from now on to the power case $f(u)=u^{p}(p>1)$ in (1.2a). Following [GP2] and [GK1], we introduce similarity variables as follows

$$
\begin{aligned}
& u(x, t)=(T-t)^{-\frac{1}{p-1}} \Phi(y, \tau), \\
& y=x(T-t)^{-1 / 2}, \tau=-\log (T-t) .
\end{aligned}
$$

We thus obtain that $\Phi$ solves

$$
\Phi_{\tau}=\Phi_{y y}-\frac{1}{2} y \Phi_{y}+\Phi^{p}-\frac{\Phi}{p-1} ; y \in \mathbb{R}, \tau>-\log T .
$$

In the new variables, $(1.3 \mathrm{a})$ reads

$$
\Phi(y, \tau) \rightarrow(p-1)^{-\frac{1}{p-1}} \quad \text { as } \tau \rightarrow \infty, \text { uniformly for }
$$

bounded $y$

Notice that $\Phi_{0}=(p-1)^{\frac{1}{p-1}}$ is a nontrivial stationary solution of (1.6). To obtain more detailed expansions, it seems natural to linearize about $\Phi_{0}$ by setting

$$
\Phi(y, \tau)=(p-1)^{-\frac{1}{p-1}}+\psi(y, \tau)
$$

and then consider the equation for the error term $\psi(y, \tau)$ which reads

$$
\begin{aligned}
& \psi_{\tau}=\psi_{y y}-\frac{y \psi_{y}}{2}+\psi+f(\psi) \equiv A \psi+f(\psi), \\
& \text { where } f(\psi)=\left((p-1)^{-\frac{1}{p-1}}+\psi\right)^{p}-(p-1)^{-\frac{1}{p-1}}-\frac{p \psi}{p-1}
\end{aligned}
$$

To analyze the evolution of $\psi(y, \tau)$ one is led to take into account the linear operator $A$ in (1.9), and this in turn motivates our choices of functional frame. For $q>1$ and $k=1,2, \ldots$ we set 
with the standard topology. We shall denote henceforth the $L_{w}^{2}$-norm by $\|\cdot\|$. The operator $A$ in (1.9) is self-adjoint in $L_{w}^{2}(\mathbb{R})$, having eigenvalues $\lambda_{n}=1-\frac{n}{2}$; $n=0,1,2, \ldots$ with eigenfunctions $H_{n}(y)$ given by

$$
H_{n}(y)=c_{n} \tilde{H}_{n}\left(\frac{y}{w}\right), \quad \text { where } \quad c_{n}=\left(2^{n / 2}(4 \pi)^{1 / 4}(n !)^{1 / 2}\right)^{-1},
$$

and $\widetilde{H}_{n}(y)$ is the standard $n^{t h}$-Hermite polynomial so that $\left\|H_{n}\right\|=1$ for any $n$.

As $\psi(y, \tau) \rightarrow 0$ when $\tau \rightarrow \infty$, it is natural to assume that the linear part will eventually dominate in (1.9). Writing $\psi(y, \tau)$ as a Fourier series.

$$
\psi(y, \tau)=\sum a_{k}(\tau) H_{k}(y)
$$

we readily see that the Fourier coefficients $a_{k}(\tau)$ satisfy

$$
\dot{a}_{k}(\tau)=\left(1-\frac{k}{2}\right) a_{k}(\tau)+\left\langle f(\psi), H_{k}\right\rangle \quad ; k=0,1,2, \ldots
$$

where $\langle$,$\rangle stands for the natural scalar product in L_{w}^{2}(\mathbb{R})$. By analogy with classical ODE theory, we would then expect that

$$
\psi(y, \tau) \sim a_{k}(\tau) H_{k}(y) \quad \text { for some } k \text { as } \tau \rightarrow \infty
$$

Indeed, not all values of $k$ are admissible in (1.12). For $k=0,1$, linearizing in (1.11b) would yield respectively

$$
\psi(y, \tau) \approx \alpha_{0} e^{\tau} H_{0}(y), \psi(y, \tau) \approx \alpha_{1} e^{\tau / 2} H_{1}(y) \quad \text { as } \quad \tau \rightarrow \infty
$$

which are incompatible with the basic assumption (1.7). For $k=3,4, \ldots$, dropping the nonlinear term in $(1.11 \mathrm{~b})$ would give

$$
\psi(y, \tau) \approx C e^{\left(1-\frac{k}{2}\right) \tau} H_{k}(y) \quad \text { as } \quad \tau \rightarrow \infty .
$$

Finally, when $k=2$ then linear part vanishes in $(1.11 \mathrm{~b})$, and we obtain

$$
\dot{a}_{2}(\tau)=\frac{1}{2} p(p-1)^{\frac{1}{p-1}} a_{2}(\tau)^{2}\left\langle H_{2}^{2}, H_{2}\right\rangle+\cdots
$$

which after integration yields

$$
(4 \pi)^{1 / 4}(p-1)^{-\frac{1}{p-1}} \cdot H_{2}(y)
$$


THEOREM 1. - Let $u(x, t)$ be a solution of (1.2), where $f$ is as in (1.3a), and assume that $u(x, t)$ blows up at $x=0, t=T$. Let $\Phi(y, \tau)$ be given in (1.5). Then one of the following cases occurs

$$
\begin{aligned}
& \Phi(y, \tau) \equiv(p-1)^{-\frac{1}{p-1}}, \\
& \Phi(y, \tau)=(p-1)^{-\frac{1}{p-1}}-\frac{\left.(4 \pi)^{1 / 4}(p-1)\right)^{-\frac{1}{p-1}}}{\sqrt{2} p} \cdot \frac{H_{2}(y)}{\tau}+o\left(\frac{1}{\tau}\right) \\
& \text { as } \tau \rightarrow \infty, \\
& \text { or } \\
& \Phi(y, \tau)=(p-1))^{-\frac{1}{p-1}}-C e^{\left(1-\frac{m}{2}\right) \tau} H_{m}(y)+o\left(e^{\left(1-\frac{m}{2}\right) \tau}\right) \\
& \text { as } \tau \rightarrow \infty, \\
& \text { where } C>0 \text { and } m \text { is an even number, } m \geq 4 .
\end{aligned}
$$

Convergence in $(2.2),(2.3)$ takes place in $H_{w}^{1}(\mathbb{R})$ as well as in $C_{\text {loc }}^{K, \alpha}(\mathbb{R})$ for any $k \geq 1$ and any $\alpha \in(0,1)$.

Concerning Theorem 1, several remarks are in order. To our knowledge, the existence of solutions behaving as in (2.2) was first conjectured in [HSS], where (2.2) was formally derived for the case $p=3$ by singular perturbation techniques. Later, a similar expansion was formally obtained in [D] for the case $f(u)=e^{u}$ in (1.1). The actual existence of solutions exhibiting the behaviour conjected in [D] has been recently shown in $[\mathrm{Br}]$, where stability properties of such solutions were also discussed. We also refer to [GP1], [BK] for further formal analysis for the power-like case. The flatter behaviours (2.3) seem to have been unnoticed until the work [GHV1], where the expansions listed in Theorem 1 were formally obtained by means of perturbative methods. On the other hand, results closely related to Theorem 1 have been simultaneously and independently obtained in [FK]. In that paper, the authors dealt with the higher-dimensional version of (1.1) with $f(u)=u^{p}, \quad p>1$, and proved that if $u(x, t)$ blows up at $x=0, t=T$, then either $\left.(\Phi(y, \tau)-(p-1))^{-\frac{1}{p-1}}\right)$ becomes exponentially small as $\tau \rightarrow \infty$, or the behaviour corresponding to (2.2) holds.

In view of our preliminary discussion in Section 1, it might be thought that Theorem 1 could be derived by a rather standard application of semilinear parabolic 
On the other hand, a quick glance at (2.2), (2.3) suggest that these expansions cannot be uniformly valid in the $y$-variable. For instance, for solutions satisfying (2.2), one has $\frac{H_{2}(y)}{\tau} \approx \frac{y^{2}}{\tau}$ for large $y$, and therefore the second and first term in the expansion become of the same order when $\frac{y^{2}}{\tau} \approx 1$. This motivates introducing in such external region the new variable $\xi=\frac{y}{\sqrt{\tau}}=\frac{x}{((T-t)|\log (T-t)|)^{1 / 2}}$. This is precisely the "ignition kernel variable", conjectured for instance in [D]. As a matter of fact, we have the following classification of possible behaviours in larger regions near blow-up (cf. [HV1], [HV2]).

THEOREM 2. Let $u(x, t), x=0$, and $t=T$ be as in Theorem 1. Then one of the following cases occurs

i) if (2.1) holds true, then $u(x, t)=((p-1)(T-t))^{-\frac{1}{p-1}}$

ii) if (2.2) holds true, then

$$
\begin{aligned}
& \lim _{t \uparrow T}(T-t)^{\frac{1}{p-1}} u\left(\xi\left((T-t) \mid(\log (T-t) \mid)^{1 / 2}, t\right)\right. \\
& =(p-1)^{-\frac{1}{p-1}}\left(1+\left(\frac{p-1}{4 p}\right) \xi^{2}\right)^{-\frac{1}{p-1}},
\end{aligned}
$$

uniformly on sets $|\xi| \leq R$ for any $R>0$.

iii) If (2.3) holds true, then

$$
\lim _{t \uparrow T}(T-t)^{\frac{1}{p-1}} u\left(\xi(T-t)^{1 / m}, t\right)=\left((p-1)\left(1+(p-1)^{\frac{p}{p-1}} C c_{m} \xi^{m}\right)^{-\frac{1}{p-1}},\right.
$$

where $C, c_{m}$ are as in (2.3) and (1.10) respectively, and convergence is uniform on sets $|x| \leq R$ for any $R>0$.

Let us give a quick idea of the main arguments behind the proof of Theorem 2. To this end, we shall specialize to the case (ii) there for definiteness. A first step consists in deriving the corresponding lower bound for $u(x, t)$

$$
\begin{aligned}
& (T-t)^{\frac{1}{p-1}} u\left(\xi((T-t)|\log (T-t)|)^{1 / 2}, t\right) \\
& \left.\geq(p-1)\left(1+\left(\frac{p-1}{4 p}\right) \xi^{2}+o(T-t)\right)\right)^{-\frac{1}{p-1}} \quad \text { as } t \uparrow T,
\end{aligned}
$$

uniformly on sets $|\xi| \leq R$ with $R>0$. 
Using (2.6), we are able to show that

$$
\|W(\cdot, \tau)-(p-1)\| \leq \frac{C}{\tau} \quad \text { as } \quad \tau \rightarrow \infty .
$$

Set now $G=W-(p-1)$. Then $G$ satisfies

$$
G_{\tau}=G_{y y}-\frac{1}{2} y G_{y}+G=\frac{p}{p-1} \cdot \frac{G_{y}^{2}}{G+(p-1)}
$$

We use variation of constants in the equation above to write $G(y, \tau)$ in the form

$$
\begin{aligned}
G(y, \tau) & \equiv \quad(\text { homogeneous term })+(\text { nonhomogeneous term }) \\
& \equiv G_{1}(y, \tau)+G_{2}(y, \tau)
\end{aligned}
$$

and prove that

$$
\lim _{\tau \rightarrow \infty} G_{1}(\xi \sqrt{\tau}, t)=\frac{(p-1)^{2} \xi^{2}}{4 p}, \text { uniformly when }|\xi| \leq C, C>0,
$$

$$
\lim _{\tau \rightarrow \infty} G_{2}(\xi \sqrt{\tau}, t)=0 \text {, uniformly when }|\xi| \leq C, C>0,
$$

whence (2.4). The more delicate part in (2.7) consists in deriving (2.7b), since this involves estimating the nonlinear term

$$
L(y, \tau) \equiv \frac{p}{p-1} \cdot \frac{G y^{2}}{G+(p-1)} \equiv C \Phi^{-(p+1)} \Phi_{y}^{2}
$$

for some $C>0$. We use (2.6) to bound above $\Phi^{-(p+1)}$. On the other hand, we show that

$$
\left|\Phi_{y}(\xi \sqrt{\tau}, \tau)\right| \leq \frac{C}{\sqrt{\tau}} \quad \text { as } \tau \rightarrow \infty, \text { uniformly for }|\xi| \leq C
$$

To obtain (2.8), we write $z=\left|\Phi_{y}\right|$, and use Kato's inequality to arrive at

$$
z_{\tau} \leq z_{y y}-\frac{y z_{y}}{z}+\left(\frac{1}{2}-\frac{p}{p-1}\right) z+p \Phi^{p-1} z
$$

This last equation is a linear one, and we may use variation of constants formula there as soon as a suitable global bound for $\Phi$ is available. As a matter of fact, we prove that 
3. Final time analysis. The results obtained in Section 2 lead naturally to the question of determining the profile of $u(x, T)$ when $x \approx 0, u(x, t)$ being a solution of the Cauchy problem under consideration which blows up at $x=0, t=T$. We then have (cf [HV3]).

THEOREM 3. Let $u(x, t), x=0$, and $t=T$ be as in Theorem 1, and assume that $u(x, t) \neq((p-1)(T-t))^{-\frac{1}{p-1}}$. Then the blow-up point $x=0$ is isolated and the following possibilities arise

a) If ii) in Theorem 2 holds true, then

$$
\lim _{x \rightarrow 0}\left(\frac{|x|^{2}}{|\log | x \mid}\right)^{-\frac{1}{p-1}} u(x, T)=\left(\frac{8 p}{(p-1)^{2}}\right)^{\frac{1}{p-1}}
$$

b) If iii) in Theorem 2 holds true, then

$$
\left.\lim _{x \rightarrow 0}\left(|x|^{\frac{m}{p-1}} \cdot u(x, T)\right)=(p-1)^{\frac{1}{p-1}+1} C c_{m}\right)^{-\frac{1}{p-1}}
$$

where $C, c_{m}$ are as in Theorem 2.

It is worth noticing that no assumption on the structure of the blow-up set was made to obtain Theorems 1 and 2. We now stress that, under our current assumptions, the blow-up set consists of isolated points. This was already known for the case where $u_{0}(x)$ is compactly supported ( $\mathrm{cf}[\mathrm{CM}]$ ) but our proof in [HV3] is different, and makes no use of lap number arguments. On the other hand, we should also mention that the upper bound corresponding to (3.1a) has been derived in [GP2] under additional assumptions on the initial value $u_{0}(x)$.

The proof of Theorem 3 proceed by means of

i) A key technical result (cf. Proposition 3.1 in [HV2]).

ii) Nondimensional scaling.

Since we believe that it might be illustrative for other situations, we shall elaborate a little bit on part ii). Suppose that (2.4) holds. We then take $\xi>0$, and consider the family of auxiliary functions

$$
\begin{aligned}
& v_{s}(x, t)=(T-s)^{\frac{1}{p-1}} u\left(\lambda(s)+x(T-s)^{1 / 2}, s+t(T-s)\right) \\
& \text { where } \lambda(s)=\xi((T-s)|\log (T-s)|)^{1 / 2} \text { and } 0<s<T,
\end{aligned}
$$

It is readily seen that

$$
\left(v_{s}\right)_{t}=\left(v_{s}\right)_{x x}+\left(v_{s}\right)^{p} \text { when } x \in \mathbb{R}, 0<t<1
$$

whereas, by (2.4) 
We now impose

$$
(T-s)^{1 / 2}|x| \leq \frac{\lambda(s)}{2} \quad, \text { i.e., }|x| \leq \frac{\xi}{2}|\log (T-s)|^{1 / 2}
$$

Then (3.5) yields

$$
\begin{aligned}
& v_{s}(x, 0) \leq\left((p-1)+\frac{(p-1)^{2}}{8 p} \xi^{2}\right)^{-\frac{1}{p-1}} \quad \text { if }(T-s) \text { is } \\
& \text { small enough, uniformly on }|x| \leq \frac{\xi}{2}|\log (T-s)|^{1 / 2} .
\end{aligned}
$$

On the other hand, by (2.9) we have that, for $T-s \leq 1$,

$$
v_{s}(x, t) \leq\left((p-1)^{-\frac{1}{p-1}}+C\left(\left|\log \left(\frac{T-s}{T}\right)\right|\right)^{-1}\right)(1-t)^{-\frac{1}{p-1}}
$$

We now argue as follows. By estimates (3.6), (3.7), we can make use of the technical result mentioned above (Proposition 3.1 in [HV2]) to obtain the following: If we consider $v_{s}(x, t)$ in a cylinder $Q_{n}=[-n, n] \times[0,1], \xi>0$ is large enough (independently on $n$ ), and $s$ is close enough to $T$, then

$$
v_{s}(x, t) \leq M_{n}<+\infty \quad \text { when }(x, t) \in Q_{n / 2} \text { uniformly as } s \uparrow T
$$

Notice that (3.8) implies that blow-up points are isolated. Indeed, setting $x=0, \tilde{x}=\lambda(s)$ and $\tilde{t}=s+t(T-s)$, we see that for $t \in[0,1]$ and $s \in\left[T-\delta, T-\frac{\delta}{2}\right]$ with $\delta>0$ small enough, $(3.8)$ provides a bound for $u(\tilde{x}, \tilde{t})$ in cylinders $S=\left\{(\tilde{x}, \tilde{t}): \quad \sigma_{1} \leq|\tilde{x}| \leq \sigma_{2}, \quad T-\frac{\delta}{2} \leq \tilde{t} \leq T\right\}$ with $\sigma_{1}>0$ and $\sigma_{1}, \sigma_{2}$ sufficiently small. Furthermore, (3.8) yields at once Schauder estimates in subcylinders $Q_{n, \delta}=\left[-\frac{n}{3}, \frac{n}{3}\right] \times[\delta, 1]$, uniformly as $s \uparrow T$ for any $\delta \in(0,1)$. This (and a typical barrier argument to control the behaviour when $t \approx 0$ ) enables us to conclude that there exists a subsequence (also denoted by $v_{s}(x, t)$ ), and a function $\bar{v}_{n}(x, t)$ such that

$$
\begin{aligned}
& v_{s}(x, t) \rightarrow \bar{v}_{n}(x, t) \text { as } s \uparrow T, \text { uniformly on } Q_{n, \delta} \text { for any } \\
& \delta \in(0,1)
\end{aligned}
$$


By allowing $n$ to go to infinity while repeating the previous argument at any step, we deduce that there exists a subsequence, still denoted by $v_{s}(x, t)$, and a function $\bar{v}(x, t)$ such that

$$
\begin{aligned}
& v_{s}(x, t) \rightarrow \bar{v}(x, t) \text { as } s \uparrow T, \text { uniformly on compact sets } \\
& \text { of } \mathbb{R} \times(0,1)
\end{aligned}
$$

$$
\bar{v}_{t}=\bar{v}_{x x}+\bar{v}^{p} \text { in } \mathbb{R} x(0,1)
$$

$$
\begin{aligned}
& \lim _{t \downarrow 0} \bar{v}(x, t)=(p-1)^{-\frac{1}{p-1}}\left(1+\left(\frac{p-1}{4 p}\right) \xi^{2}\right)^{-\frac{1}{p-1}}, \\
& \bar{v}(x, t) \leq M(1-t)^{-\frac{1}{p-1}} \quad \text { for some } M>0 .
\end{aligned}
$$

From all the statements contained in (3.10), only (3.10d) is new with respect to (3.9). Actually, (3.10d) holds for any solution of (3.4) which blows up at $t=1$ and has smooth and bounded initial values (cf [GP2]), and this last restriction is easily removed by application of classical parabolic theory.

It then follows from (3.10) that $\bar{v}(x, t)$ is unique, and

$$
\bar{v}(x, t)=(p-1)^{-\frac{1}{p-1}}\left((1-t)+\left(\frac{p-1}{4 p}\right) \xi^{2}\right)^{-\frac{1}{p-1}}
$$

We are ready now to conclude the proof of (3.1). We just make use of (3.10a) and (3.11) to write

$$
v_{s}(0,1)=\left(\frac{4 p}{(p-1)^{2}}\right)^{-\frac{1}{p-1}} \xi^{-\frac{2}{p-1}}+o(1) \quad \text { as } \quad s \uparrow T
$$

i.e.,

$$
\begin{aligned}
& (T-s)^{\frac{1}{p-1}} u\left(\xi((T-s)|\log (T-s)|)^{1 / 2}, t\right)=\left(\frac{4 p}{(p-1)^{2}}\right)^{-\frac{1}{p-1}} \xi^{-\frac{2}{p-1}}+ \\
& +o(1) \quad \text { as } \quad s \uparrow T
\end{aligned}
$$


whence

$$
T-s \approx\left(y\left(\sqrt{2} \xi(|\log | y||)^{1 / 2}\right)^{-1}\right)^{2} \quad \text { as } \quad s \uparrow T
$$

which, after substitution in (3.12), yields

$$
\left(\frac{|y|}{\sqrt{2}(|\log | y||)^{1 / 2}}\right)^{\frac{2}{p-1}} u(y, T) \approx\left(\frac{4 p}{(p-1)^{2}}\right)^{\frac{1}{p-1}} \quad \text { as } y \rightarrow 0
$$

which is the desired result. The proof of (3.2) is similar; cf. [HV3] for details.

We refer to [HV1] and [HV4] cf. also [BB]) for results alike to Theorems 1-3 when $f(u)=e^{u}$ in (1.1).

4. On the occurrence of different blow-up behaviours. The results in Section 2 and 3 provide a classification of all possible behaviours of the solution $u(x, t)$ of (1.2) when $f(u)=u^{p}, p>1$, near a blow-up point. However, the question of the actual existence of solutions exhibiting such behaviours has not been addressed yet. A close look at Theorem 1 reveals that, if $u(x, t)$ blows up as indicated in (2.2) (resp. as indicated in (2.3) there) then a single maximum in the scaled variable $y$ arrives to the blow-up point $y=0$ as $\tau \rightarrow \infty$ (resp. exactly $\left(\frac{m}{2}\right)$ maxima arrive to $y=0$ as $\tau \rightarrow \infty)$. This is a consequence of the very structure of the Hermite polynomials

This suggests at once that the different blow-up behaviours listed in Sections 2 and 3 depend on the number of maxima which collapse exactly at blow-up. As a matter of fact, we have

TheOREM 4. Let $u(x, t), x=0$ and $t=T$ be as in Theorem 1. Then there holds 
Let us remark briefly on Theorem 4. Part a) is quite natural, since the number of maxima of solutions of parabolic equations cannot increase in time. This basic fact has been repeatedly and independently used by many authors; cf. for instance $[\mathrm{M}],[\mathrm{A}],[\mathrm{AF}], \ldots .$. As to $\mathrm{b}$ ), we need to obtain a solution which has two maxima collapsing at blow-up. To this end, we proceed by considering initial values $u_{0, R}(x)$ consisting of two symmetric bumps a distance $R$ apart,

As $R>0$ varies, intuition suggests that

i) For $R$ small there is blow-up at a single point,

ii) For $R$ large there is blow-up at two points.

Taking the infimum of such $R$ for which (ii) above holds we obtain a value $R^{*}$ such that the corresponding solution arrives at $(0, T)$ with two maxima. This is the natural candidate for the initial value in part b) in Theorem 4, and this is the way we proceeded to derive such a result. However, we should point out that a rigorous proof needs to overcome some technical problems. In particular we need to establish

Continuity of the blow-up time with respect to the initial data, Continuity of the location of blow-up points with respect to the initial values

While (4.1) is comparatively easy, (4.2) requires in our approach of rather sophisticated techniques (in particular, we rely on Proposition 3.1 in [HV2], which was already an ingredient in the proof of Theorem 3). See also [GK3] for a related argument. Similar results hold when $f(u)=e^{u}$ in (1.2); cf. [HV4].

One may expect solutions satisfying $(2.3)$ to exist for any value $m=6,8,10, \ldots$ There would be characterized by collapse of $3,4,5, \ldots$ maxima at blow-up. We have been unable to prove existence of such solutions so far. 
Let $R>0$ be given, and let $u(x, t)$ be a positive solution of

$$
u_{t}=u_{x x}+u^{p} \quad \text { when }=-R<x<R, t>0,
$$

with $p>1$, such that

$$
u(x, 0)=u_{0}(x) \text { when }-R<x<R \text {, where } u_{0}(x) \text { is as in }
$$

Notice that no boundary conditions are prescribed. It is first shown in $[\mathrm{V}]$ that all our previous analysis carries through if blow up occurs at the interior of $(-R, R)$. More precisely, we have

THEOREM 5. Let $u(x, t)$ be a solution of (5.1), (5.2) which blows up at $x=\bar{x} \in(-R, R)$ and $t=T$. Assume that the blow-up set is contained in an interval $[-\delta, \delta]$ with $0<\delta<R$. Then the asymptotic behaviour of $u(x, t)$ as $(x, t) \rightarrow(\bar{x}, T)$ is given by Theorem 1 (with $y=(x-\bar{x})(T-t)^{-1 / 2}$ in $(1.4 b)$ ). Moreover, the results of Theorems 2 and 3 also hold true.

We point out that the separation assumption on the blow-up set made above is known to hold in many cases, as for instance,

When homogeneous Dirichlet (or Neumann) conditions are added to $(5.1),(5.2)$,

In general, whenever the number of maxima is a priori bounded.

(cf. $[\mathrm{FM}],[\mathrm{GK} 1],[\mathrm{CM}], \ldots$ ). Actually, violation of (5.3b) leads to an example in which new behaviours arise and the blow-up set reaches the boundary.

THEOREM 6. For any $R>0$, there exists $\delta>0$ small enough and a solution $\widetilde{u}(x, t)$ of the equation

$$
u_{t}=u_{x x}+u^{p} \quad \text { in }(-R, R) \times(T-\delta, T), p>1
$$

such that $\widetilde{u}(x, t) \neq((p-1)(T-t))^{-\frac{1}{p-1}}$ and $\widetilde{u}$ blows up in the whole interval $(-R, R) \times(T-\delta, T)$. Moreover, we have that

$$
\begin{aligned}
& \left|\widetilde{u}(x, t)(T-t)^{\frac{1}{p-1}}-(p-1)^{-\frac{1}{p-1}}-(T-t)^{-1} w(i x, T-t)\right| \\
& \leq K \exp \left(-\frac{5}{4}(T-t)^{-2}\right)
\end{aligned}
$$


the number of maxima increases as $t \uparrow T$. In particular, if $t_{1}<t_{2}<T$ we have a situation as that depicted below.

6. General blow up patterns. Let us return now to the study of the Cauchy problem considered in Sections 2 to 4. So far, we have obtained a classification of all possible blow-up behaviours, and have shown that the $H_{2}(y)$ and $H_{4}(y)$ patterns actually occur (cf. Theorem 4). We have also seen that it is reasonable to expect the existence of $H_{m}(y)$ behaviours for $m=6,8,10, \ldots$ although no proof of such fact is known to us as yet. We next set out to examine the following question

which of these behaviours is likely to be observed $\Gamma$

or, in another words

which of the previous behaviours is stable under small perturbations $\Gamma$

Again, it is easy to guess what the logical answer should be. Indeed, the $H_{2}(y)$ pattern in (2.2) looks stable, whereas those in (2.3) do not. The reason is that any $H_{m}(y)$ behaviour $(m=4,6,8, \ldots)$ would correspond to $\left(\frac{m}{2}\right)$ maxima coalescing exactly at blow up, a delicate balance likely to be destroyed by a slight change in the initial value $u_{0}(x)$. Any such change however, would lead to a new datum still having one maximum if that was the case for the original value $u_{0}(x)$, and this strongly indicates the stability of the $H_{2}(y)$-profile.

We next proceed to state these results in a precise way. Let $\widetilde{u}(x, t)$ be a solution 
and assume that

$$
\widetilde{u}(x, t) \text { blows up at points } x_{1}, \ldots, x_{k} \quad(k \geq 1) \text { at time } t=T \text {. }
$$

We then have

THEOREM 7. a) Suppose that $\widetilde{u}_{0}(x)$ is continuous, nonnegative and compactly supported, $\tilde{u}_{0}(x) \in C_{0}(\mathbb{R})$. Then, for any $\varepsilon>0$ there exists $u_{0}(x) \in C_{0}(\mathbb{R})$ such that

$$
\max _{x \in \mathbb{R}}\left|u_{0}(x)-\tilde{u}_{0}(x)\right| \leq \varepsilon
$$

and the solution $u(x, t)$ of (6.1) with initial value $u_{0}(x)$ blows up at a single point $\bar{x}$ and (2.2) (with $y=(x-\bar{x})(\bar{T}-t)^{-1 / 2}, \bar{T}$ being the new blow up time) holds. Moreover, $u_{0}(x)$ can be selected so that, for any fixed $i=1, \ldots, k$

$$
\left|x_{1}-\bar{x}\right| \rightarrow 0 \quad \text { as } \quad \varepsilon \rightarrow 0
$$

b) Suppose now that $\tilde{u}(x, t)$, blows up at a single point $\tilde{x}$ and (2.2) (with $\left.\left.y=(x-\tilde{x})(T-t)^{-1 / 2}\right)\right)$ holds. Then there exists $\varepsilon>0$ small enough, depending on $\tilde{u}_{0}(x)$, such that for any $u_{0}(x) \in C_{0}(\mathbb{R})$ which satisfies (6.3), the corresponding solution $u(x, t)$ blows up at a single point $\bar{x}$, and (2.2) (with $y=(x-\bar{x})(\bar{T}-t)^{-1 / 2}$ ) holds. Moreover (6.4) is also satisfied with $x_{1}$ replaced by $\tilde{x}$. In another words

$$
\begin{aligned}
\text { Generic blow-up } \equiv & \text { Single point blow-up of } H_{2}(y) \text {-type, i.e., } \\
& \text { such that (2.4) holds }
\end{aligned}
$$

Before proceeding any further, a few remarks are in order. We do not really need $\tilde{u}_{0}(x)$ to be compactly supported. All that is required is blow-up to occur in a compact set, and this indeed happens if $\tilde{u}_{0}(x)$ decays rapidly enough at infinity (cf. [GK3]). On the other hand, the existence of solutions which blow up at exactly $k$ points (with $k$ arbitrary) has been recently proved in [M] for Dirichlet problems in bounded intervals; cf. in this context our discussion in Section 5 above. Finally, generic properties have been discussed for higher-dimensional versions of (1.1) in some cases. Consider for instance the problem

$$
\begin{array}{ll}
u_{t}=\Delta u+e^{u} & \text { when } x \in \Omega, t>0 \\
u(x, 0)=u_{0}(x) & \text { for } x \in \Omega, t=0, \\
u(x, t)=0 & \text { for } x \in \partial \Omega, \tau \geq 0
\end{array}
$$


Theorem 7 is proved in [HV5]. We shall here restrict ourselves to stress the main ideas in the proof of part a). As indicated by the very nature of the result, our approach is a perturbative one. Suppose that $\tilde{u}(x, t)$ solves $(6.1),(6.2)$. We set

$$
u_{0}(x)=u_{0, \varepsilon}(x)=\widetilde{u}_{0}(x)+\varepsilon \widetilde{R}_{0}(x)
$$

where $0<\varepsilon<1$, and $\widetilde{R}_{0}(x)$ will be selected later. Let $u_{\varepsilon}(x, t)$ be the solution of (6.1) with initial value $u_{0}(x) \mathrm{s}$ in (6.3). Then, formally

$$
u_{\varepsilon}(x, t)=\widetilde{u}(x, t)+\varepsilon \widetilde{R}(x, t)+\cdots
$$

where $\widetilde{R}$ satisfies

$$
\begin{aligned}
& \widetilde{R}_{t}=\widetilde{R}_{x x}+p \tilde{u}^{p-1} \widetilde{R} \\
& \widetilde{R}(x, 0)=\widetilde{R}_{0}(x)
\end{aligned}
$$

Notice that $(6.5 \mathrm{a})$ is linear, but the potential $p \widetilde{u}^{p-1}$ becomes singular at $t=T$. A key point in the proof consists in deriving the following result

Let $x_{1}$ be a blow-up point of $\tilde{u}$. Then, for $i=1, \ldots, k$

$$
\widetilde{R}(x, t) \approx \alpha_{1}(T-t)^{-\frac{p}{p-1}} \quad \text { uniformly on sets }\left|x-x_{1}\right| \leq C(T-1)^{1 / 2}
$$

where the $\alpha_{1}$ are generically arbitrary. More precisely, for any given set of constants $\beta_{1}, \ldots, \beta_{k}$, and any $\delta>0$, we may pick $\widetilde{R}_{0}(x)$ such that $(6.6)$ holds with $\left|\alpha_{1}-\beta_{1}\right|<\delta$ for any $i=1, \ldots, k$.

Consider now the perturbed solution $u_{\varepsilon}(x, t)$ (cf. (6.4)). By (1.3a), we know that

$$
u_{\varepsilon}(x, t)=\left((p-1)\left(T_{\varepsilon}-t\right)\right)^{-\frac{1}{p-1}}+\cdots
$$

$T_{\varepsilon}$ being the new blow-up time. On the other hand, by (6.4) and (6.6), we have that

$$
u_{\varepsilon}(x . t)=(p-1)(T-t)^{-\frac{1}{p-1}}+\varepsilon \alpha_{1}(T-t)^{-\frac{p}{p-1}}+\cdots
$$


we deduce from such a result that

$$
\Delta T_{\varepsilon} \approx-\varepsilon \alpha_{1}(p-1)^{-\frac{p}{p-1}}
$$

Once (6.6) and (6.9) have been obtained, our strategy goes as follows. We pick one of the blow-up points of $\tilde{u}$, say $x_{j}$, and select then $\widetilde{R}_{0}(x)$ so that (6.6) holds with $\alpha_{1}<0$ when $i \neq j$ and $\alpha_{j}>0$. Recalling (6.9), blow-up is postponed near $x_{i}(i \neq j)$ and it is anticipated near $x_{j}$. Since the number of maxima of $\tilde{u}$ is finite by our assumptions on $\widetilde{u}_{0}(x)$, repetition of the previous argument leads to the situation where there is a single point blow-up at, say, $x=0$ with perhaps several maxima collapsing there. For simplicity, we shall just consider the case of two maxima, so that the rescaled space profile looks like

We then derive a refinement of (6.6). Namely, we prove that $\widetilde{R}_{0}(x)$ can be selected such that

$$
\begin{aligned}
& \widetilde{R}(x, t) \approx \alpha x(T-t)^{-\frac{p}{p-1}}, \text { uniformly on sets } \\
& |x| \leq C(T-t)^{1 / 2}, \text { where } \alpha \neq 0
\end{aligned}
$$

Recalling (6.8) and (2.3), we obtain

$$
u_{\varepsilon}(x, t)=((p-1)(T-t))^{-\frac{1}{p-1}}-C(T-t)^{-\frac{1}{p-1}+1} H_{4}(y)
$$


$x(t)$ is the level line described by the maximum located to the left of $x=0$, we see that

$$
u_{\varepsilon}(x(t), t)<((p-1)(T-t))^{-\frac{1}{p-1}} \quad \text { for } \quad t \approx T
$$

Since $M(t)=u_{\varepsilon}(x(t), t)$ satisfies $M(t) \leq M(t)^{p}$, it follows from (6.11) that $x(t)$ cannot reach the blow up point at blow up time, whence the desired result. The case where $\alpha<0$ is similar

7. Related evolution problems. The approach sketched above applies to a variety of problems of the type

$$
u_{t}=u_{x x} \pm f(u), \quad f \text { nonnegative }
$$

For instance, it can be used to analyze the extinction or dead core case $([\mathrm{K}]$, $[\mathrm{EK}],[\mathrm{BS}],[\mathrm{FH}],[\mathrm{CMM}],[\mathrm{GHV} 2], \ldots)$. Consider the Cauchy problem

$$
\begin{array}{ll}
u_{t}=u_{x x}-u^{p} & \text { when } x \in \mathbb{R}, t>0,0<p<1 \\
u(x, 0)=u_{0}(x) & \text { when } x \in \mathbb{R},
\end{array}
$$

where $u_{0}(x)$ is as in (1.2b). It is known that the solution $u(x, t)$ of $(7.1),(7.2)$ vanishes in a finite time, in the sense that there exists $T^{*} \in(0, \infty)$ such that $u(x, t) \equiv 0$ for $t \geq T^{*}$. The infimum of such times $T^{*}$ is then called the extinction time $T$ of $u$. A point $x_{0}$ is named an extinction point if there exist sequences $\left\{x_{n}\right\},\left\{t_{n}\right\}$ such that $\lim _{n \rightarrow \infty} x_{n}=x_{0}, \lim _{n \rightarrow \infty} t_{n}=T$ and $u\left(x_{n}, t_{n}\right)>0$ for any $n$.

On the other hand, solutions may develop dead cores, i.e., regions where $u(x, t)=$ 0 for $t<T$, even when $u_{0}(x)$ is everywhere positive. The sets where $u>0$ and $u=0$ are separated by interfaces or free boundaries. The following results have been proved in [HV6].

THEOREM 8. Assume that $u_{0}(x)$ is as in (1.2b) and has a single maximum. Then $u(x, t)$ vanishes at some time $t=T$ at a single extinction point $x_{0}$. Moreover, we have

$$
\begin{aligned}
& \left.\lim _{t \uparrow T}(T-t)^{-\frac{1}{p-1}} u\left(x_{0}+\xi((T-t))|\log (T-t)|\right)^{1 / 2}, t\right) \\
& =(1-p)^{-\frac{1}{p-1}}\left(1-\left(\frac{1-p}{4 p}\right) \xi^{2}\right)_{+}^{-\frac{1}{p-1}}
\end{aligned}
$$




$$
\lim _{t \uparrow T} \frac{\xi_{1}^{2}(t)}{(T-t)|\log (T-t)|}=\left(\frac{4 p}{1-p}\right) \quad \text { for } \quad i=1,2
$$

The reader will notice the analogy between (7.3) and (2.4), the role of (1.4a) being played now by the explicit solution

$$
\bar{u}(x, t)=((1-p)(T-t))_{+}^{-\frac{1}{p-1}}
$$

However interfaces cannot appear in (1.2), and need to be dealt with by means of new suitable techniques in the extinction case. On the other hand, as in (1.2), flatter asymptotics are indeed possible. In particular, we have

THEOREM 9. There exists an initial value $u_{0}(x)$ and a constant $C>0$ such that the corresponding solution of (7.1), (7.2) has a single extinction point at $x=x_{0}, t=T$, and satisfies

$$
\begin{aligned}
& \lim _{t \uparrow T}(T-t)^{-\frac{1}{p-1}} u\left(x_{0}+\xi(T-t)^{1 / 4}, t\right) \\
& =(1-p)^{\frac{1}{p-1}}\left(1-C \xi^{4}\right)_{+}^{\frac{1}{p-1}}
\end{aligned}
$$$$
\text { uniformly on sets }|\xi| \leq R \quad \text { with } \quad R>0 \text {. }
$$

Moreover, there exist continuous curves $S_{1}(t), S_{2}(t)$ such that, for $t \approx T$,

$$
u(x, t)>0 \text { in } I=\left(x_{0}-\delta_{1}(t), x_{0}+\delta_{2}(t)\right), u(x, t)=0 \text { if } x \notin 1 .
$$

$$
\lim _{t \uparrow T} \frac{S_{i}(t)}{(T-t)^{1 / 4}}=\left(\frac{1}{C}\right)^{1 / 4} \quad \text { for } \quad i=1,2, \quad \text { where } C \text { is as in (7.5). }
$$

When $p<0$ in (7.1), the absorption term there becomes singular when $u=0$, and one is led to the so-called quenching problems (usually written in the variable $v=1-u$ ), which have been extensively studied after reference [Ka] appeared; see for instance [Le2] for a comprehensive survey on that problem. An interesting question there is that of describing the asymptotic profile of solutions near quenching points, i.e., near points where $u$ becomes zero. We refer to $[\mathrm{FG}]$ for recent results in such direction. 
[Be] H. Bellout, A criterion for blow-up of solutions to semilinear heat equations, SIAM J. Math. Anal. 18 (1987), 722-727.

[BB] J. Bebernes and S. Bricher, Final time blow up profiles for semilinear parabolic equations via center manifold theory, SIAM J. Math Anal., to appear.

[BBE] J. Bebernes, A. Bressan and D. Eberly, A description of blow-up for the solid fuel ignition model, Indiana Univ. Math. J. 36 (1987), 131-136.

[BE] J. Bebernes and D. Eberly, Mathematical problems from combustion theory, Applied Mathematical Sciences v. 83, Springer Verlag (1989).

[BK] M. Berger and R.V. Kohn, A rescaling algorithm for the numerical calculation of blowing up solutions, Comm. Pure Appl. Math. 41 (1988), 841-863.

[BS] C. BandLe and I. Stakgold, The formation of the dead core in a parabolic reaction-diffusion equation, Trans. Amer. Math. Soc. 286 (1984), 275-293.

[Br1] A. BRessan, On the asymptotic shape of blow-up, Indiana Univ. Math. J. to appear.

[Br2] A. Bressan, Stable blow-up patterns, J. Diff. Equations, to appear.

[CF] L.A. Caffarelit and A. Friedman, Blow-up of solutions of nonlinear heat equations, J. Math. Anal. and Appl. 129 (1988), 409-419.

[CM] X.Y. Chen and H. Matano, Convergence, asymptotic periodicity and finite blow-up in one-dimensional semilinear heat equations, J. Diff. Equations, 78 (1989), 160-190.

[CMM] X. Chen, H. Matano and M. Mimura, Finite-point extinction and continuity of interfaces in a semilinear diffusion equation with strong absorption, to appear.

[D] J. Dold, Analysis of the early stage of thermal runaway, Quart. J. Mech. appl. Math. 38 (1985), 361-387.

[EK] L.C. Evans and B.F. KNERR, Instantaneous shrinking of the support of nonnegative solutions to certain nonlinear parabolic equations and variational inequalities, Ill. J. Math. 23 (1979), 153-166.

[FG] S. Filippas AND J.S. Guo, Quenching profiles for one-dimensional semilinear heat equations, to appear.

[FK] S. Filippas and R.V. Kohn, Refined asymptotics for the blow-up of $u_{t}-\Delta u=u^{p}$, Comm. Pure Appl. Math., to appear,.

[FM] A. FRIEDMAN AND J.B. MC LEOD, Blow-up of positive solutions of semilinear heat equations, Indiana Univ. Math. J. 34 (1985), 425-447.

[Fu] H. FuJita, On the blowing-up of solutions of the Cauchy problem for $u_{t}=\Delta u+A u^{1+\alpha}, \mathrm{J}$. Fac. Sci. Univ. of Tokyo, Section I, 13 (1966), 109-124.

[GHV1] V.A. Galaktionov, M.A. Herrero and J.J.L. Velázquez, The space structure near a blow-up point for semilinear heat equations: a formal approach, USSR Comp. Math. and Math. Physics, 31, 3 (1991), 399-411.

[GHV2] V.A. Galaktionov, M.A. Herrero and J.J.L. Velázquez, The structure of solutions near an extinction point in a semilinear heat equation with strong absorption: a formal approach, To appear in the volume Nonlinear diffusion equations and their equilibrium states. N.G. Lloyd et al. ed., Birkhäuser Verlag.

[GP1] V.A. Galaktionov and S.A. Posashkov, The equation $u_{t}=u_{x x}+u^{\beta}$. Localization, asymptotic behaviour of unbounded solutions, Preprint N. 97 M., Keldysh Inst. Appl. Math. (1985) (in russian).

[GP2] V.A. Galaktionov and S.A. Posashkov, Application of new comparison theorems in the investigation of unbounded solutions of nonlinear parabolic equations, Diff. Urav. 22, 7 (1986), 1165-1173. 
[H] D. Henry, Geometric theory of semilinear parabolic equations, Springer Lecture Notes in Mathematics No. 840 (1981).

[VI1] M.A. Herrero and J.J.L. Velázquez, Blow up behaviour of one dimensional semilinear parabolic equations, Ann. Inst. Henri Poincaré, to appear.

[HV2] M.A. Herrero and J.J.L. VelázQuez, Flat blow-up in one-dimensional semilinear heat equations, Differential and Integral Equations, to appear.

[HV4] M.A. Herrero and J.J.L. Velázquez, Plane structures in thermal runaway, Israel J. Maths., to appear.

[HV5] M.A. Herrero and J.J.L. Velázquez, Generic behaviour of one-dimensional blow-up patterns, Annali della Sc. Norm. Sup. de Pisa, to appear.

[HV6] M.A. Herrero and J.J.L. Velázquez, Approaching an extinction point in one-dimensional semilinear heat equations with strong absorption, J. Math. Anal. and Appl. to appear.

[HSS] L.M. Hocking, K. Stewartson and J.T. Stuart, A nonlinear instability burst in plane parallel flow, J. Fluid Mech. 51 (1972), 705-735.

$[\mathrm{K}]$ A.S. Kalashnikov, The propagation of disturbances in problems of nonlinear heat conduction with strong absorption, USSR. Comp. Math. and Phys. 14 (1974), 70-85.

[Ka] H. KAWARADA, On solutions of initial boundary values problems for $u_{t}=u_{x x}+\frac{1}{1-u}$, RIMS Kyoto U. 10 (1975), 729-736.

[L] A.A. LACEY, Mathematical analysis of thermal runaway for spatially inhomogeneous reactions, SIAM J. Appl. Math. 43 (1983), 1350-1386.

[Le1] H.A. Levine, Some nonexistence and instability theorems for solutions of formally parabolic equations of the form $u_{t}=-A u+F(u)$, Arch. Rat. Mech. Anal. 51,5 (1973), 371-386.

[Le2] H.A. LEvine, Quenching, nonquenching and beyond quenching for solutions of some parabolic equations, Annali di Mat. Pura ed Appl. CLV (1989), 243-250.

[M] H. Matano, Nonincrease of the lap number of a solution for a one-dimensional semilinear parabolic equation, J. Fac. Sci, Univ. Tokyo, Sec. IA, 29 (1982), 401-441.

[Me] F. MERLE, On the existence of a blow-up solution of the nonlinear heat equation with arbitrarily given blow-up points, to appear.

[V] J.J.L. Velízquez, Local behaviour near blow-up points for semilinear parabolic equation, J. Diff. Equations, to appear.

[W] F.B. Weissler, Single point blow-up of semilinear initial value problems, J. Diff. Equations 55 (1984), 204-224.

[Wi] D.V. Widder, The heat equation, Academic Press, New-York (1975). 